$\underline{\text { Preprint typeset in JHEP style. - HYPER VERSION }}$

DISTRIBUTION A.

hep-ph/9903464

MIT-CTP-2841

Revised April 28, 1999

\title{
Day-Night and Energy Dependence of MSW Solar Neutrinos for Maximal Mixing
}

\author{
Alan H. Guth, Lisa Randall, Mario Serna \\ guth@ctp.mit.edu,randall@baxter.mit.edu,mariojr@mit.edu \\ Center for Theoretical Physics \\ Massachusetts Institute of Technology \\ Cambridge, Massachusetts 02139, U.S.A.
}

\begin{abstract}
It has been stated in the literature that the case of maximal mixing angle for $\nu_{e}$ leads to no day-night effect for solar neutrinos and an energy independent flux suppression of $\frac{1}{2}$. While the case of maximal mixing angle and $\Delta m^{2}$ in the MSW range does lead to suppression of the electron neutrinos reaching the earth from the sun by $P_{S}=\frac{1}{2}$, the situation is different for neutrinos that have passed through the earth. We make the pedagogical point that, just as with smaller mixing angles, the earth regenerates the $\left|\nu_{1}\right\rangle$ state from the predominantly $\left|\nu_{2}\right\rangle$ state reaching the earth, leading to coherent interference effects. This regeneration can lead to a day-night effect and an energy dependence of the suppression of solar electron neutrinos, even for the case of maximal mixing. For large mixing angles, the energy dependence of the day-night asymmetry depends heavily on $\Delta m^{2}$. With a sufficiently sensitive measurement of the day-night effect, this energy dependence could be used to distinguish among the large mixing angle solutions of the solar neutrino problem.
\end{abstract}

KEYWORDS: solar neutrinos, neutrino oscillations, MSW, day-night effect, earth regeneration, bi-maximal mixing, maximal mixing, energy independent flux suppression. 


\section{Contents}

1. Introduction 1

2. Derivation of Equation (1.1) 3

3. The day-night effect at maximal mixing

4. Conclusions 8

A. Derivation of MSW equations 9

B. Calculation methodology for the day-night effect 13

C. Validity of the steady state approximation

D. The zenith distribution function 17

\section{Introduction}

The recent Super-Kamiokande announcement that atmospheric neutrinos are nearly maximally mixed has renewed much interest in the possibility that solar neutrinos might also be maximally mixed. In this paper we will consider only two-neutrino mixings, so by "maximal mixing" we are referring to the possibility that the two lightest mass eigenstates, $\left|\nu_{1}\right\rangle$ and $\left|\nu_{2}\right\rangle$, with eigenvalues $m_{1}$ and $m_{2}$ respectively $\left(m_{1}<m_{2}\right)$, are each equal-probability superpositions of the flavor eigenstate $\left|\nu_{e}\right\rangle$ (electron neutrino) and some other state $\left|\nu_{x}\right\rangle$, where $\left|\nu_{x}\right\rangle$ can be any linear combination of $\left|\nu_{\mu}\right\rangle$ (muon neutrino) and $\left|\nu_{\tau}\right\rangle$ (tau neutrino). Many theoretical models have been proposed predicting the possibility of such maximal mixing (for exam-

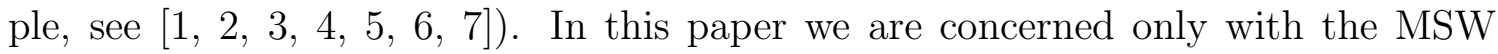
solutions to the solar neutrino problem, first proposed by Mikheyev, Smirnov, and Wolfenstein [8, 9, 10], while the alternative possibility of nearly maximally mixed vacuum oscillations has been considered by other authors [11. The MSW effect results from the neutrino interaction with matter, causing an enhancement of the conversion process transforming $\nu_{e}$ into $\nu_{x}$. The MSW effect is also capable of driving neutrinos back towards a $\nu_{e}$ state after passing through the earth. This process would result in a change in the $\nu_{e}$ flux between daytime and nighttime measurements, a phenomenon known as the day-night effect, or more generally zenith angle 
dependence. Over the past decade there have been extensive studies of the day-night effect [12, 13, 14, 15, 16, 17, 18, 19, which have been mostly concerned with the small mixing angle solutions to the solar neutrino problem. Most of these studies have used the Mikheyev-Smirnov expression [20] to describe the effect of the earth on the solar neutrinos, which we will hereafter refer to as Eq. (1.1):

$$
P_{S E}=\frac{P_{S}-\sin ^{2} \theta_{V}+P_{2 e}\left(1-2 P_{S}\right)}{\cos 2 \theta_{V}} .
$$

Here $P_{S}$ is the probability that an electron neutrino $\left(\left|\nu_{e}\right\rangle\right)$ originating in the sun will be measured as an electron neutrino upon reaching the earth, $P_{S E}$ is the probability that an electron neutrino originating in the sun will be measured as an electron neutrino after passing through the earth, $P_{2 e}$ is the probability that a pure $\left|\nu_{2}\right\rangle$ eigenstate entering the earth will be measured as an electron neutrino when it emerges, and $\theta_{V}$ is the vacuum mixing angle, defined by

$$
\begin{aligned}
& \left|\nu_{1}\right\rangle=\left|\nu_{e}\right\rangle \cos \theta_{V}-\left|\nu_{x}\right\rangle \sin \theta_{V}, \\
& \left|\nu_{2}\right\rangle=\left|\nu_{x}\right\rangle \cos \theta_{V}+\left|\nu_{e}\right\rangle \sin \theta_{V} .
\end{aligned}
$$

In the previous studies of the day-night effect several authors have claimed that there is no day-night effect at $P_{S}=1 / 2$ [18, 14]. In fact, in the formula for the daynight effect which is conventionally used, Eq. (1.1), the properties of the earth enter only through $P_{2 e}$, which is explicitly multiplied by $\left(1-2 P_{S}\right)$. We wish to emphasize, however, that the case of maximal mixing is an exception to this statement. For maximal mixing Eq. (1.1) is ill-defined, since $\cos 2 \theta_{V}=0$, and we will show below that generically there is a day-night effect for this case. Nonetheless, we have no disagreements with either the equations or the contour plots in the aforementioned papers, which in fact do show non-zero day-night effects at maximal mixing. The purpose of this paper is to clarify the previous papers, and also to investigate more carefully the role of the day-night effect for maximal mixing. We will show that at maximal mixing $P_{S E} \neq 1 / 2$, implying a day-night effect and an often overlooked energy-dependence of the suppression of the solar neutrino flux.

Physically, the day-night effect survives because the neutrino beam reaching the earth, for all MSW solutions, is predominantly $\left|\nu_{2}\right\rangle$. For maximal mixing this state is half $\nu_{e}$ and half $\nu_{x}$, but there is a definite phase relationship, $\left|\nu_{2}\right\rangle=\left(\left|\nu_{e}\right\rangle+\left|\nu_{x}\right\rangle\right) / \sqrt{2}$, so the density matrix is not proportional to the identity matrix. A coherent component of $\left|\nu_{1}\right\rangle$ is regenerated as this beam traverses the earth, leading to interference with the incident $\left|\nu_{2}\right\rangle$ beam. The case is rather different from the small mixing-angle case, for which Eq. (1.1) really does imply the absence of a day-night effect when $P_{S}=1 / 2$. For a small mixing angle $P_{S}$ equals $1 / 2$ only when conditions in the sun drive the 
ensemble into a density matrix proportional to the identity matrix, in which case the earth would have no effect.

In the remainder of this paper we explain in more detail why maximal mixing can result in a day-night effect. In Section 2, we review the derivation of Eq. (1.1) as given by Mikheyev and Smirnov [20], and we resolve the maximal mixing ambiguity. Next in Section 3 we present results of numerical calculations showing the day-night effect at maximal mixing. Finally in the appendices, we provide greater details concerning the analytic and numerical calculations presented in the paper.

\section{Derivation of Equation (1.1)}

The key assumption necessary for the derivation of Eq. (1.1) is that the neutrino beam arriving at the earth can be treated as an incoherent mixture of the two mass eigenstates $\left|\nu_{1}\right\rangle$ and $\left|\nu_{2}\right\rangle$. That is, we assume that there is no interference between the $\nu_{1}$ and $\nu_{2}$ components reaching the earth, or equivalently that the off-diagonal entries of the density matrix in the $\nu_{1}-\nu_{2}$ basis are negligibly small. The physical effects which cause this incoherence are discussed in Appendix Q. In the case of maximal mixing, the incoherence is ensured for $\Delta m^{2}>6.5 \times 10^{-9} \mathrm{eV}^{2}$ because of the energy resolution of current detectors. Other sources of incoherence include the separation of $\left|\nu_{1}\right\rangle$ and $\left|\nu_{2}\right\rangle$ wave packets in transit to the earth, the averaging over the regions in the sun where the neutrinos were produced, and the averaging over the changing radius of the earth's orbit [21]. In Appendix 9 we comment on the regions of parameter space for which the assumption of incoherence is valid.

Given the assumption of incoherence, we write the fractions of $\left|\nu_{1}\right\rangle$ and $\left|\nu_{2}\right\rangle$ flux from the sun as $k_{1}$ and $k_{2}$, respectively ${ }^{1}$. Since there is no interference, the probability that a solar neutrino will be measured as $\nu_{e}$ upon reaching the surface of the earth is given by

$$
\begin{aligned}
P_{S} & =k_{1}\left|\left\langle\nu_{e} \mid \nu_{1}\right\rangle\right|^{2}+k_{2}\left|\left\langle\nu_{e} \mid \nu_{2}\right\rangle\right|^{2} \\
& =k_{1} \cos ^{2} \theta_{V}+k_{2} \sin ^{2} \theta_{V} \\
& =\cos ^{2} \theta_{V}-k_{2} \cos 2 \theta_{V},
\end{aligned}
$$

where we have used Eqs. (1.2) and the fact that $k_{1}+k_{2}=1$. Similarly, the probability that a solar neutrino will be measured as $\nu_{e}$ after passing through the earth, when it is no longer in an incoherent superposition of the mass eigenstates, is given by

$$
P_{S E}=k_{1} P_{1 e}+k_{2} P_{2 e}
$$

where $P_{1 e}\left(P_{2 e}\right)$ is the probability that a $\left|\nu_{1}\right\rangle\left(\left|\nu_{2}\right\rangle\right)$ eigenstate will be measured as $\nu_{e}$ after traversing the earth. Finally, the unitarity of the time evolution operator

\footnotetext{
${ }^{1}$ For large mixing angles, $\sin ^{2} 2 \theta_{V} \geq 0.5$ and $5 \times 10^{-5} \leq \Delta m^{2}(\mathrm{eV})^{2} \leq 1 \times 10^{-7}, k_{2} \approx 1$ and $k_{1} \approx 0$.
} 
implies that the state vectors of two neutrinos entering the earth as $\left|\nu_{1}\right\rangle$ and $\left|\nu_{2}\right\rangle$ must remain orthonormal as they evolve through the earth and become $\left|\tilde{\nu}_{1}\right\rangle$ and $\left|\tilde{\nu}_{2}\right\rangle$, respectively. Therefore

$$
P_{1 e}+P_{2 e}=\left|\left\langle\nu_{e} \mid \tilde{\nu}_{1}\right\rangle\right|^{2}+\left|\left\langle\nu_{e} \mid \tilde{\nu}_{2}\right\rangle\right|^{2}=1 .
$$

Eq. (1.1) can then be obtained by using Eq. (2.1) and the above equation to eliminate $P_{1 e}, k_{1}$, and $k_{2}$ from Eq. (2.2).

From the above derivation, one can see that the singularity of Eq. (1.1) at maximal mixing arises when Eq. (2.1) is solved to express $k_{2}$ in terms of $P_{S}$. For maximal mixing $P_{S}=1 / 2$ for any value of $k_{2}$, so $k_{2}$ cannot be expressed in terms of $P_{S}$. The

ambiguity disappears, however, if one leaves $k_{2}$ in the answer, so Eq. (2.3) can be used to rewrite Eq. (2.2) as

$$
P_{S E}=\frac{1}{2}+2\left(k_{2}-\frac{1}{2}\right)\left(P_{2 e}-\frac{1}{2}\right) .
$$

Thus, $P_{S E}=1 / 2$ only if $k_{2}=1 / 2$ or $P_{2 e}=1 / 2$. For the MSW solutions at maximal mixing one has $k_{2} \approx 1$, and there is no reason to expect $P_{2 e}=1 / 2$. Generically $P_{S E} \neq 1 / 2$ for the case of maximal mixing.

\section{The day-night effect at maximal mixing}

Using the evolution equations derived in Appendix $\mathrm{A}$ and the procedures described in Appendix B, we have calculated a variety of properties concerning the day-night effect for maximal mixing angle. The calculation parameters are chosen for those of the Super-Kamiokande detector.

Figure 11 shows the evolution of $P\left(\nu_{e} \rightarrow \nu_{e}\right)$, the probability that a solar neutrino will be measured as $\nu_{e}$, as the beam of neutrinos traverses a path through the center of the earth. Notice that after traversing the earth the ensemble of neutrinos is no longer in a steady state, but instead $P\left(\nu_{e} \rightarrow \nu_{e}\right)$ continues to oscillate in the vacuum. From the perspective of the mass eigenstates, the neutrinos under consideration arrive at the earth roughly in a $\left|\nu_{2}\right\rangle$ state. Upon reaching the earth, the step-function-like changes in the electron density profile (see Fig. 5i) cause nonadiabatic evolution, regenerating the $\left|\nu_{1}\right\rangle$ state and leading to interference effects. In the regions of parameter space where the day-night effect is maximal because the oscillation length of these interference terms coincides with the length of the slabs of near constant density composing the earth, the resulting buildup of $\nu_{e}$ flux has been called oscillation length resonance [22, 23, 24].

In Fig. 2 we present a contour plot calculated from the density matrix that exemplifies the non-zero nature of the day-night effect at maximal mixing. On the left is a three-dimensional surface where the height of the surface is the day-night asymmetry. Notice that the exposed edge is calculated at maximal mixing and is 


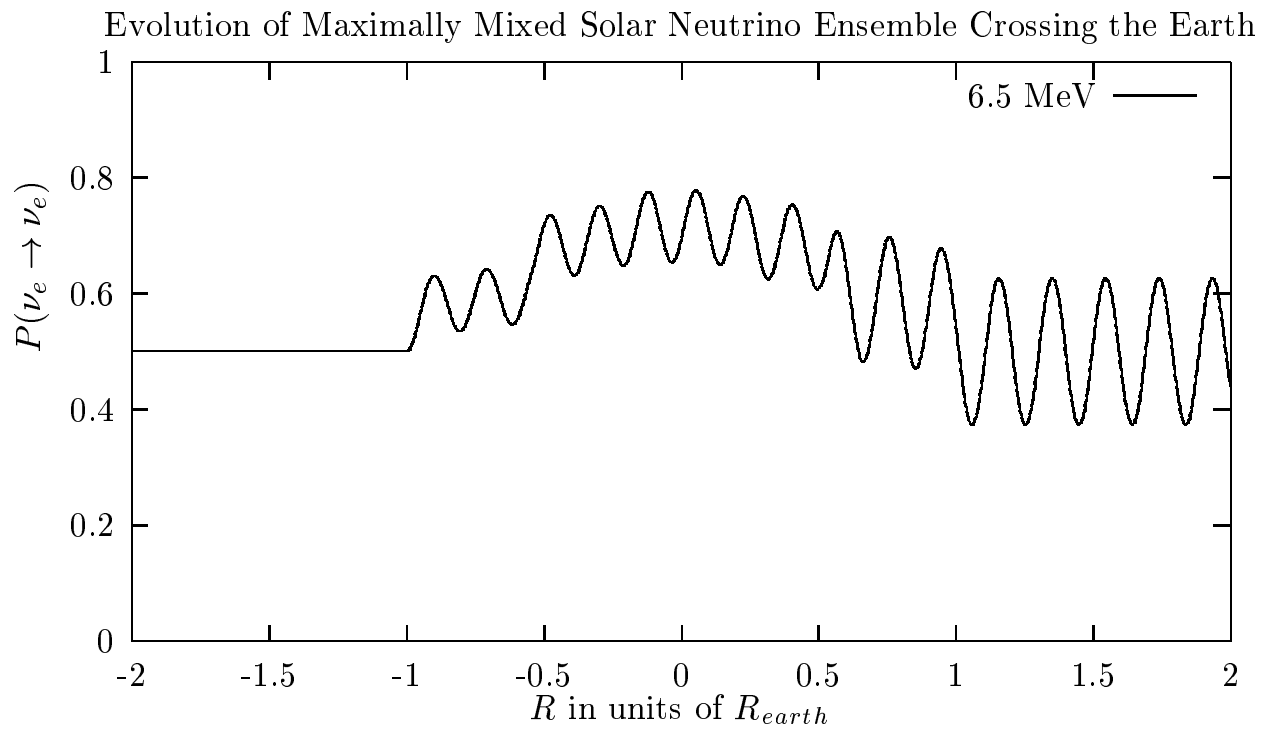

Figure 1: The evolution of $P_{\nu_{e} \rightarrow \nu_{e}}$ as the ensemble of neutrinos propagates across the center of the earth. The neutrinos enter the earth as an incoherent mixture of the energy eigenstates $\nu_{1}$ and $\nu_{2}$ which is almost completely $\nu_{2}$. This plot shown is for $\Delta m^{2}=$ $1.3 \times 10^{-5} \mathrm{eV}^{2}$ and a neutrino energy $E=6.5 \mathrm{MeV}$.
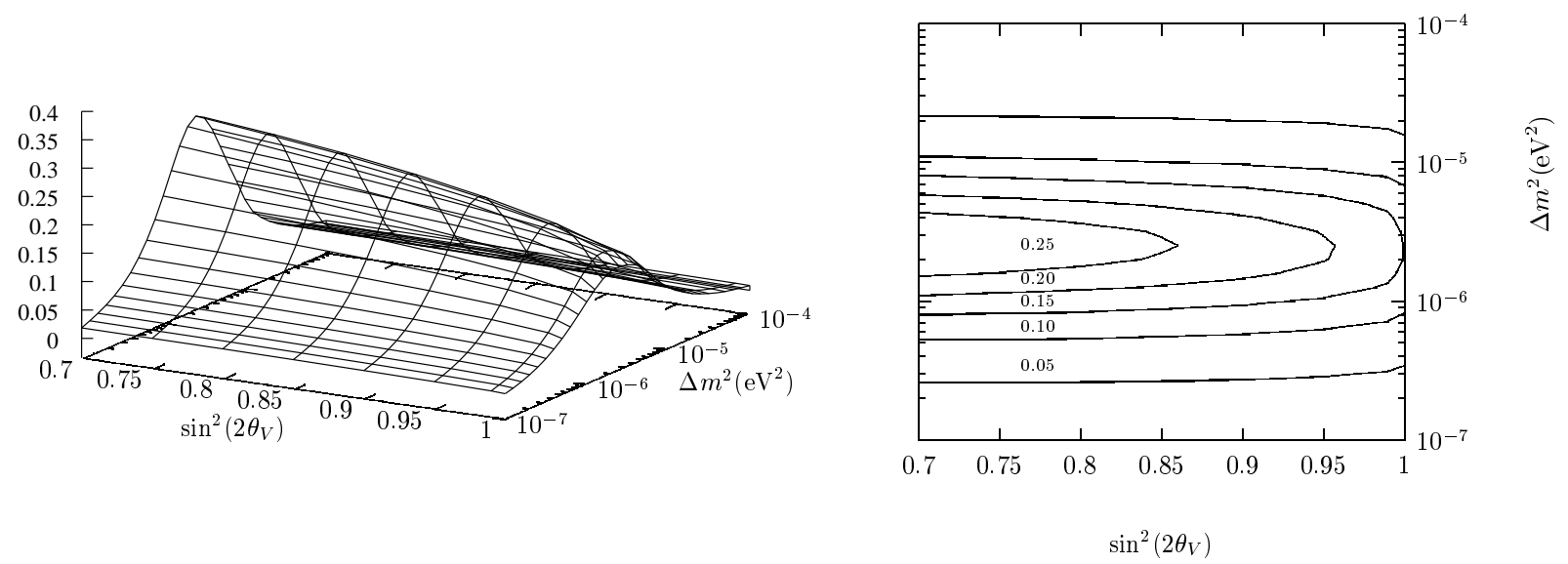

Figure 2: The day-night asymmetry $\left(A_{d-n}=(N-D) /(N+D)\right)$ as a function of mixing parameters calculated using the density matrix. On the left is a three dimensional surface where the height of the surface is the day-night asymmetry. Notice that the exposed edge is calculated at maximal mixing and is clearly non-zero. On the right is a contour plot showing the lines of constant day-night asymmetry.

clearly non-zero. On the right is a contour plot showing the lines of constant daynight asymmetry, a plot which is identical to those produced in other references.

We now explain how the inclusion of the day-night effect at maximal mixing resolves a certain confusion that has arisen in the past because of its neglect. As a result of the non-zero day-night effect, there exists an energy dependence at maximal 


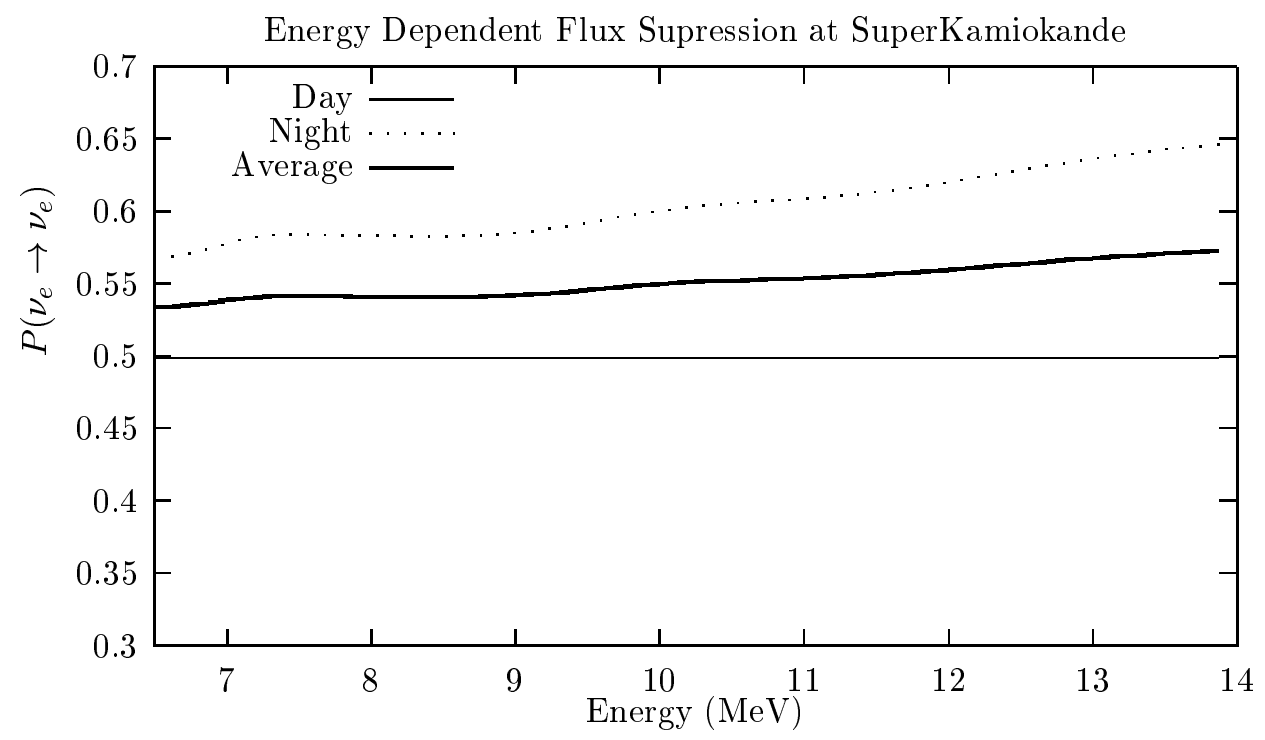

Figure 3: The predicted flux suppression as a function of energy. Notice that the predicted overall flux suppression is not $1 / 2$, due to day-night effects, even though the mixing angle is maximal. The plot is for $\Delta m^{2}=1.0 \times 10^{-5} \mathrm{eV}^{2}$ which is near the border of the region excluded by the small day-night effect $\left(A_{d-n}\right)$ measured at Super-Kamiokande.

mixing, as can be seen in Fig. 3. If one assumes that the flux suppression at maximal mixing has no energy dependence, as was done in Ref. [25], then there is an apparent discrepancy between two sections of Ref. [26]. Sec. IV-D excludes the possibility of energy-independent oscillation into active (as opposed to sterile) neutrinos at the 99.8\% confidence level, while Fig. 2 shows some regions of the maximal-mixingangle parameter space not excluded at the $99 \%$ confidence level. Ref. 225 has tried to resolve this discrepancy without including the day-night effect, concluding that maximal mixing is excluded at the $99.6 \%$ confidence level. The actual resolution to this apparent discrepancy is that Fig. 2 of Ref. [26] includes the energy dependence induced by the day-night effect at maximal mixing, while Sec. IV-D discusses the case of energy-independent flux suppression and does not apply to maximal mixing. The correct conclusion is that of Fig. 2, which shows that maximal mixing is not excluded at the $99 \%$ confidence level.

Whether or not the day-night effect is included, maximal mixing is not a very good fit to the experimental data from the three neutrino experiments (chlorine, gallium, and water) 26]. However, maximal mixing does fit well if the chlorine data is excluded on the suspicion of some systematic error [27]. Ref. [3] has argued that if the ${ }^{8} B$ flux is about $17 \%$ lower than the standard solar model (BP98) 28], then a bimaximal mixing scenario becomes a tenable solution to the solar neutrino problem. The MSW mechanism described here is applicable for $\Delta m^{2}>6.5 \times 10^{-9} \mathrm{eV}^{2}$. In the bi-maximal mixing scenario that we consider the upper bound on $\Delta m^{2}$ is set by the CHOOZ data constraining $\Delta m^{2} \leq 0.9 \times 10^{-3} \mathrm{eV}^{2}$ [29]. 


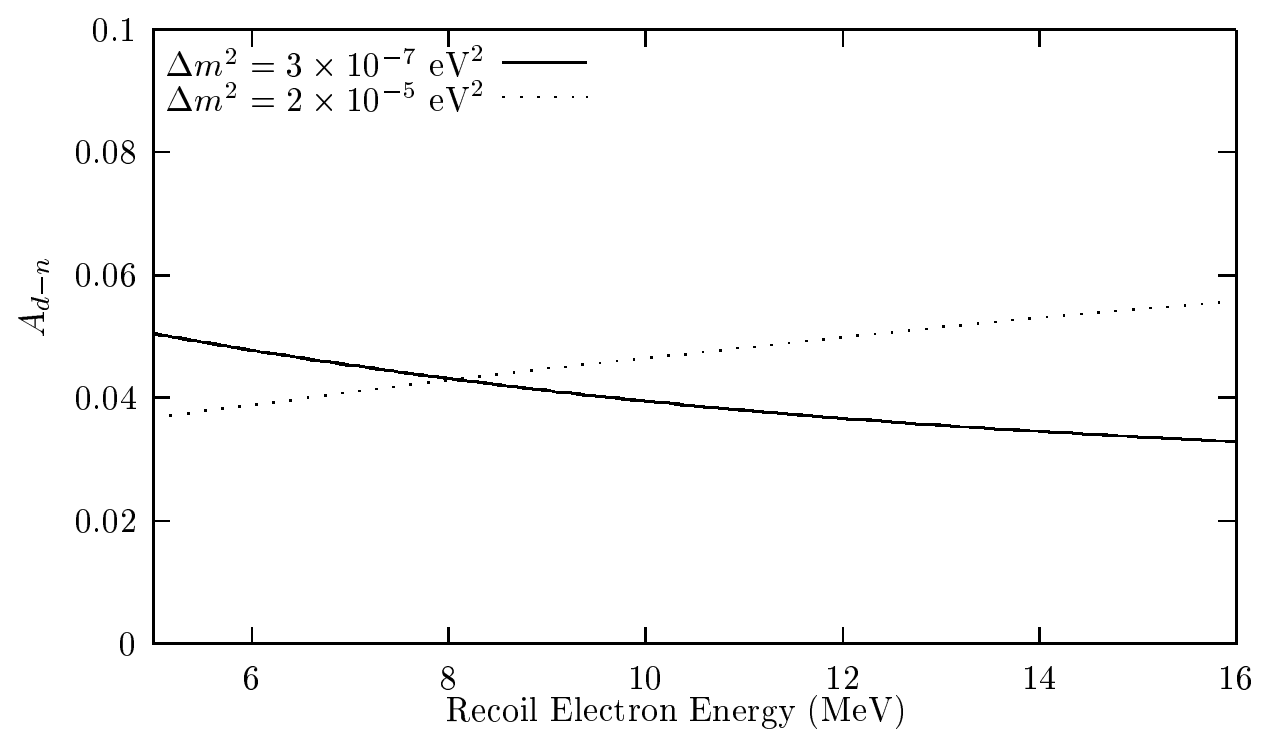

Figure 4: The day-night asymmetry $\left(A_{d-n}=(N-D) /(N+D)\right)$ as a function of recoil electron energy at Super-Kamiokande. Both plots are at maximal mixing angle, with $\Delta m^{2}$ at the upper and lower borders of the region disfavored by the smallness of the day-night effect observed at Super-Kamiokande. The rising line is for $\Delta m^{2}=2 \times 10^{-5} \mathrm{eV}^{2}$, and the descending line is for $\Delta m^{2}=3 \times 10^{-7} \mathrm{eV}^{2}$.

When detailed studies of the day-night effect are completed, the energy (and zenith angle) dependence will be valuable additional information. To the best of our knowledge, the Super-Kamiokande Collaboration has not published their daynight asymmetry as a function of recoil electron energy. Past studies of the daynight effect have noted the energy dependence of the day-night asymmetry [17, 18]. While for small mixing angles $\left|A_{d-n}\right|<0.02$ without a clear energy dependence [18], for large mixing angles the $A_{d-n}$ energy dependence can be significant and informative. Fig. $⿴$ shows the theoretical predictions of the day-night asymmetry in the electron recoil spectrum at Super-Kamiokande for two cases of maximal mixing: $\Delta m^{2}=2 \times 10^{-5} \mathrm{eV}^{2}$ and $\Delta m^{2}=3 \times 10^{-7} \mathrm{eV}^{2}$. Note that the two curves have opposite slopes.

The approximate shape of the graph of $A_{d-n}$ vs. recoil electron energy can be understood from Fig. 2, using the fact that Fig. 2 is dominated by the peak of the ${ }^{8} B$ neutrino spectrum at about $6.5 \mathrm{MeV}$. It is shown in Appendix $\mathrm{A}$ that the neutrino evolution equations (Eqs. (A.5)-( $\mathrm{A.8})$ ) depend on $\Delta m^{2}$ and the neutrino energy (or momentum) $E$ only through the combination $\Delta m^{2} / E$. Thus, Fig. 2 shows that for any value of $\sin ^{2} 2 \theta_{V}, A_{d-n}$ has a maximum at $\Delta m^{2} / E \approx 2.5 \times 10^{-6} \mathrm{eV}^{2} /(6.5 \mathrm{MeV})$. When $E$ is varied at fixed $\Delta m^{2}, A_{d-n}$ will have a peak at

$$
E \approx \frac{\Delta m^{2}}{2.5 \times 10^{-6} \mathrm{eV}^{2}} \times 6.5 \mathrm{MeV} .
$$

So for $\Delta m^{2}=2 \times 10^{-5} \mathrm{eV}^{2}$ the peak lies far to the right of the scale in Fig. 4 , so the 
curve slopes upward. For $\Delta m^{2}=3 \times 10^{-7} \mathrm{eV}^{2}$ the peak lies far to the left, and the curve slopes downward.

Fig. 2 shows that the peak in the graph of $A_{d-n}$ vs. $\Delta m^{2}$ is higher at large mixing angles $\left(\sin ^{2} 2 \theta_{V} \approx 0.7\right)$ than it is at maximal mixing, so the same will be true for the energy dependence of the day-night effect. For $\sin ^{2} 2 \theta_{V}=0.63$ and $\Delta m^{2}=1.3 \times 10^{-5} \mathrm{eV}^{2}$, for example, the slope of the graph of $A_{d-n}$ vs. recoil electron energy is about twice the magnitude of the slopes shown in Fig. 4 . Thus, the daynight asymmetry as a function of recoil electron energy could be a strong indicator of $\Delta m^{2}$ if the solar neutrinos have a large or maximal mixing angle in the MSW range of parameters.

\section{Conclusions}

We have disproved the assumption that $P_{S}=1 / 2$ always implies $P_{S E}=1 / 2$. We have also shown that neutrinos with a maximal mixing angle can have a day-night effect and that they do not always result in a uniform energy-independent flux suppression of $1 / 2$. Because the issues that we have attempted to clarify concern mainly the words that have been used to describe correct equations (which were generally used numerically), there are no changes to most constraints presented in other references. The only corrections apply to fits of energy-independent suppressions; that is the fits no longer apply to the exclusion of some regions of maximally mixed neutrinos. Finally, we have noted that the energy dependence of the day-night effect can be a strong discriminator between various solutions of the solar neutrino problem.

\section{Acknowledgments}

We would like to thank Paul Schechter for a useful conversation. We also greatly appreciate Kristin Burgess, Yang Hui He, and Jun Song for reviewing the paper. MS would like to thank the National Science Foundation (NSF) for her gracious fellowship, and the Air Force Institute of Technology (AFIT) for supporting this research. MS would like to thank Dr. Krastev for helping us understand the parameters involved in calculating the contour plots for the day-night effect. We would also like to thank Robert Foot for useful comments on the manuscript. This work is sup-

ported in part by funds provided by the U.S. Department of Energy (D.O.E.) under cooperative research agreement \#DF-FC02-94ER40818. 


\section{A. Derivation of MSW equations}

In this appendix we present a derivation of the MSW effect. First we derive the MSW equations of motion for an individual neutrino. We then find the energy eigenstates of the system and use them to find the wave function amplitudes for electron neutrinos produced in the sun and evolved into the vacuum. To describe the ensemble of neutrinos we introduce the density matrix. After averaging out the rapid oscillations we find a steady state solution to the density matrix equations of motion. We average this solution over the regions of neutrino production.

We begin by finding the MSW equations of motion for an individual neutrino. The coupling describing the interaction between electron neutrinos and electrons is

$$
H_{\text {int }}=\sqrt{2} G_{F} N_{e}
$$

where $N_{e}$ is the number density of electrons. This contribution to the interaction Hamiltonian is added to the Schrödinger equation written in the flavor basis. We assume that $\left|\nu_{e}\right\rangle$ can be written as a superposition of only two mass eigenstates, $\left|\nu_{1}\right\rangle$ and $\left|\nu_{2}\right\rangle$. We let $\left|\nu_{x}\right\rangle$ denote the orthogonal linear combination of $\left|\nu_{1}\right\rangle$ and $\left|\nu_{2}\right\rangle$, which might be any superposition of $\left|\nu_{\mu}\right\rangle$ (muon neutrino) and $\left|\nu_{\tau}\right\rangle$ (tau neutrino). The transformation between the $\nu_{1}-\nu_{2}$ and $\nu_{e}-\nu_{x}$ bases is then given by

$$
\left(\begin{array}{l}
C_{\nu_{1}} \\
C_{\nu_{2}}
\end{array}\right)=\left(\begin{array}{cc}
\cos \theta_{V} & -\sin \theta_{V} \\
\sin \theta_{V} & \cos \theta_{V}
\end{array}\right)\left(\begin{array}{l}
C_{\nu_{e}} \\
C_{\nu_{x}}
\end{array}\right)
$$

where the variable $\theta_{V}$ is the vacuum mixing angle, and $C_{\nu} \equiv\langle\nu \mid \Psi\rangle$ for $\nu=\nu_{1}, \nu_{2}, \nu_{e}$ or $\nu_{x}$. This equation can be written compactly by introducing the index notation

$$
C_{\nu_{i}}=U_{i f}^{\dagger} C_{\nu_{f}}
$$

where the repeated index $f$ is summed over $\nu_{e}$ and $\nu_{x}$, and $i$ is summed over the mass eigenstates. The Schrödinger equation for this system is:

$$
i \partial_{t}\left(\begin{array}{l}
C_{\nu_{e}} \\
C_{\nu_{x}}
\end{array}\right)=\left[U\left(\begin{array}{cc}
p+\frac{m_{1}^{2}}{2 p} & 0 \\
0 & p+\frac{m_{2}^{2}}{2 p}
\end{array}\right) U^{\dagger}+\left(\begin{array}{cc}
\sqrt{2} G_{F} N_{e} & 0 \\
0 & 0
\end{array}\right)\right]\left(\begin{array}{l}
C_{\nu_{e}} \\
C_{\nu_{x}}
\end{array}\right)
$$

where we have expanded the energy in the ultra-relativistic limit so that $E=p+\frac{m^{2}}{2 p}$. We now substitute $U$ into the Schrödinger equation, obtaining

$$
i \partial_{t}\left(\begin{array}{l}
C_{\nu_{e}} \\
C_{\nu_{x}}
\end{array}\right)=\left(\begin{array}{cc}
B & A \\
A & -B
\end{array}\right)\left(\begin{array}{l}
C_{\nu_{e}} \\
C_{\nu_{x}}
\end{array}\right)
$$

where

$$
\begin{aligned}
\Delta_{0} & \equiv \frac{1}{2 p}\left(m_{2}^{2}-m_{1}^{2}\right) \\
A & \equiv \frac{\Delta_{0}}{2} \sin 2 \theta_{V} \\
B & \equiv \frac{\sqrt{2}}{2} G_{F} N_{e}-\frac{\Delta_{0}}{2} \cos 2 \theta_{V},
\end{aligned}
$$


and where we have dropped the term $p+\frac{\left(m_{1}^{2}+m_{2}^{2}\right)}{4 p}+\frac{\sqrt{2}}{2} G_{F} N_{e}$ which is proportional to the identity, because terms proportional to the identity cannot contribute to mixing.

The eigenvalues are $\pm \lambda\left(N_{e}\right)$, where $\lambda\left(N_{e}\right)=\sqrt{A^{2}+B^{2}}$, and the eigenvectors are:

$$
v_{-}=\left(\begin{array}{c}
\sqrt{\frac{\lambda-B}{2 \lambda}} \\
-\sqrt{\frac{\lambda+B}{2 \lambda}}
\end{array}\right), \text { and } v_{+}=\left(\begin{array}{l}
\sqrt{\frac{\lambda+B}{2 \lambda}} \\
\sqrt{\frac{\lambda-B}{2 \lambda}}
\end{array}\right) .
$$

Since these eigenvectors form the matrix that will diagonalize the interaction matrix in the presence of matter, it is useful to parameterize them by a matter mixing angle $\theta_{M}\left(N_{e}\right)$ :

$$
\cos \theta_{M}=\sqrt{\frac{\lambda-B}{2 \lambda}}, \text { and } \sin \theta_{M}=\sqrt{\frac{\lambda+B}{2 \lambda}}
$$

or equivalently

$$
\lambda \cos 2 \theta_{M}=-B
$$

or

$$
\lambda \sin 2 \theta_{M}=A .
$$

Defining the matrix

$$
U\left(\theta_{M}\right)=\left(\begin{array}{cc}
\cos \theta_{M} & \sin \theta_{M} \\
-\sin \theta_{M} & \cos \theta_{M}
\end{array}\right),
$$

the Hamiltonian can be diagonalized as

$$
U\left(\theta_{M}\right)\left(\begin{array}{cc}
-\lambda & 0 \\
0 & \lambda
\end{array}\right) U^{\dagger}\left(\theta_{M}\right)=\left(\begin{array}{cc}
B & A \\
A & -B
\end{array}\right) .
$$

We maintain the notation introduced in Eq. (A.3) so that $C_{\nu_{i}}\left(\theta_{M}\right)=U_{i f}^{\dagger}\left(\theta_{M}\right) C_{\nu_{f}}$ in or out of matter, where $C_{\nu_{i}}\left(\theta_{M}\right) \equiv\left\langle\nu_{i} \mid \Psi\right\rangle$ denotes the amplitude for the overlap of the neutrino state with instantaneous mass eigenstates $\left|\nu_{i}\right\rangle$.

To describe the evolution of the neutrinos as they travel to the earth from their creation point in the sun, it is useful to develop the adiabatic approximation, in which one assumes that the density changes imperceptibly within an oscillation length. Remembering that $U, \theta_{M}$, and $\lambda$ are all functions of the local electron density $N_{e}$, and hence functions of time, we write the Schrödinger equation in the basis $\nu_{i}\left(\theta_{M}\right)$ of the instantaneous mass eigenstates:

$$
\begin{aligned}
i \partial_{t}\left(\begin{array}{l}
C_{\nu_{1}} \\
C_{\nu_{2}}
\end{array}\right) & =\left(\begin{array}{cc}
-\lambda & 0 \\
0 & \lambda
\end{array}\right)\left(\begin{array}{l}
C_{\nu_{1}} \\
C_{\nu_{2}}
\end{array}\right)+\left(i \partial_{t} U^{\dagger}\right) U\left(\begin{array}{c}
C_{\nu_{1}} \\
C_{\nu_{2}}
\end{array}\right) \\
& =\left(\begin{array}{cc}
-\lambda & 0 \\
0 & \lambda
\end{array}\right)\left(\begin{array}{l}
C_{\nu_{1}} \\
C_{\nu_{2}}
\end{array}\right)+i\left(\begin{array}{cc}
0 & -\partial_{t} \theta_{M} \\
\partial_{t} \theta_{M} & 0
\end{array}\right)\left(\begin{array}{l}
C_{\nu_{1}} \\
C_{\nu_{2}}
\end{array}\right) .
\end{aligned}
$$

The adiabatic approximation is the assumption that the off-diagonal terms $\partial_{x} \theta_{M}$ can be neglected, in which case the equation is easily integrated:

$$
\left(\begin{array}{l}
C_{\nu_{1}}\left(t_{f}\right) \\
C_{\nu_{2}}\left(t_{f}\right)
\end{array}\right) \approx\left(\begin{array}{cc}
e^{+i \phi\left(t_{f}\right)} & 0 \\
0 & e^{-i \phi\left(t_{f}\right)}
\end{array}\right)\left(\begin{array}{l}
C_{\nu_{1}}\left(t_{0}\right) \\
C_{\nu_{2}}\left(t_{0}\right)
\end{array}\right)
$$


where

$$
\phi\left(t_{f}\right)=\int_{t_{0}}^{t_{f}} \lambda(t) d t
$$

Because the adiabatic states form a complete basis, we can always write the exact solution as a superposition of the two adiabatic states. This final superposition is expressed by two unknown variables, $a_{1}$ and $a_{2}$ where $\left|a_{1}\right|^{2}+\left|a_{2}\right|^{2}=1$. The $\left|a_{2}\right|^{2}$ parameter represents the probability of a non-adiabatic transition, which is most likely to happen when the neutrinos cross resonance, the density at which $B=0$, when the two eigenvalues become nearly equal. Likewise $\left|a_{1}\right|^{2}=1$ would represent adiabatic evolution. Given any initial state $\nu_{f}\left(t_{0}\right)$ in the flavor basis, the final state can be written in the general form:

$$
\left(\begin{array}{l}
C_{\nu_{1}}\left(t_{f}\right) \\
C_{\nu_{2}}\left(t_{f}\right)
\end{array}\right)=\left(\begin{array}{cc}
a_{1} & a_{2} \\
-a_{2}^{*} & a_{1}^{*}
\end{array}\right)\left(\begin{array}{cc}
e^{+i \phi\left(t_{f}\right)} & 0 \\
0 & e^{-i \phi\left(t_{f}\right)}
\end{array}\right) U^{\dagger}\left(\theta_{M}\left(t_{0}\right)\right) \nu_{f}\left(t_{0}\right) .
$$

For an electron neutrino originating in a medium of mixing angle $\theta_{M}$, the above equation implies that the final state in the vacuum is given by

$$
\left(\begin{array}{l}
C_{\nu_{1}}\left(t_{f}\right) \\
C_{\nu_{2}}\left(t_{f}\right)
\end{array}\right) \equiv\left(\begin{array}{c}
A_{1} \\
A_{2}
\end{array}\right)=\left(\begin{array}{c}
a_{1} \cos \theta_{M} e^{+i \phi}+a_{2} \sin \theta_{M} e^{-i \phi} \\
-a_{2}^{*} \cos \theta_{M} e^{+i \phi}+a_{1}^{*} \sin \theta_{M} e^{-i \phi}
\end{array}\right) .
$$

We now go on to talk about the ensemble of neutrinos reaching the earth. To describe a quantum mechanical ensemble of neutrinos, it is useful to introduce the density matrix

$$
\rho \equiv \sum_{i} f_{i}\left|\nu_{i}\right\rangle\left\langle\nu_{i}\right|
$$

where $f_{i}$ denotes the probability that the particle is in the quantum state $\left|\nu_{i}\right\rangle$. The density matrix corresponding to a single neutrino as described by Eq. (A.20) is therefore given by

$$
\rho=\left(\begin{array}{cc}
\left|A_{1}\right|^{2} & A_{1} A_{2}^{*} \\
A_{1}^{*} A_{2} & \left|A_{2}\right|^{2}
\end{array}\right)
$$

where

$$
\begin{aligned}
& \left|A_{1}\right|^{2}=\frac{1}{2}\left[1+\cos 2 \theta_{M}\left(1-2\left|a_{2}\right|^{2}\right)\right]+\frac{1}{2}\left[a_{1} a_{2}^{*} \sin 2 \theta_{M} e^{2 i \phi\left(t_{f}\right)}+c . c\right] \\
& A_{1} A_{2}^{*}=\frac{1}{2} \sin 2 \theta_{M}\left[a_{1}^{2} e^{2 i \phi\left(t_{f}\right)}-a_{2}^{2} e^{-2 i \phi\left(t_{f}\right)}\right]-a_{1} a_{2} \cos 2 \theta_{M} \\
& \left|A_{2}\right|^{2}=\frac{1}{2}\left[1-\cos 2 \theta_{M}\left(1-2\left|a_{2}\right|^{2}\right)\right]-\frac{1}{2}\left[a_{1} a_{2}^{*} \sin 2 \theta_{M} e^{2 i \phi\left(t_{f}\right)}+c . c\right]
\end{aligned}
$$

In Appendix Q we explain why this process allows us to eliminate the terms that have rapidly oscillating phases. In particular, the phase angle $\phi\left(t_{f}\right)$ and the phases of the complex numbers $a_{1}$ and $a_{2}$ are all rapidly varying functions of the neutrino energy, the location in the sun where the neutrino is produced, and the precise time of day and year at which the neutrino is observed. The density matrix which describes the 
ensemble of observed neutrinos is constructed by averaging over these quantities, so any quantity with a rapidly oscillating phase will average to zero. This is equivalent to the statement that the $\nu_{1}$ and $\nu_{2}$ components arriving at the earth are incoherent, so we average over their phases. The matrix elements of the phase-averaged density matrix are given by

$$
\begin{aligned}
\left\langle\left|A_{1}\right|^{2}\right\rangle & =\frac{1}{2}\left[1+\cos 2 \theta_{M}\left(1-2\left|a_{2}\right|^{2}\right)\right] \\
\left\langle A_{1} A_{2}^{*}\right\rangle & =0 \\
\left\langle\left|A_{2}\right|^{2}\right\rangle & =\frac{1}{2}\left[1-\cos 2 \theta_{M}\left(1-2\left|a_{2}\right|^{2}\right)\right] .
\end{aligned}
$$

The term $\left|a_{2}\right|^{2} \equiv P_{\text {jump }}$ is the probability of crossing from one adiabatic state to the other during the time evolution of these operators. An approximate expression for $P_{\text {jump }}$ can be found by using a linear approximation for the density profile at resonance [30], yielding

$$
P_{\text {jump }}=\exp \left(-\frac{\pi \Delta m^{2} \sin ^{2}\left(2 \theta_{V}\right) N\left(x_{\mathrm{res}}\right)}{4 p \cos \left(2 \theta_{V}\right) N^{\prime}\left(x_{\mathrm{res}}\right)}\right) .
$$

Here $N\left(x_{\text {res }}\right)$ is the density at the point where the neutrino crosses resonance, and $N^{\prime}\left(x_{\text {res }}\right)$ is the first derivative of the density at resonance. More accurate approximations to $P_{\text {jump }}$ and the details of their derivation can be found in Refs. [31, 32] and the references therein.

The density matrix corresponding the ensemble of observed neutrinos must be obtained by averaging over the production sites in the sun. While we have already made use of this fact in dropping all terms with rapidly oscillating phases, we must still average the slowly varying terms which remain. Letting ${ }^{8} B(r)$ denote the normalized probability distribution for production at a distance $r$ from the center of the sun, one finds

$$
\rho=\left(\begin{array}{cc}
\frac{1}{2}+C_{0} & 0 \\
0 & \frac{1}{2}-C_{0}
\end{array}\right)
$$

where

$$
C_{0}=\frac{1}{2} \int_{0}^{R_{\text {sun }}} d r^{8} B(r) \cos \left(2 \theta_{M}(r)\right)\left(1-2 P_{\text {jump }}\right) .
$$

Note that the diagonal entries of $\rho$ are just the fractions $k_{1}$ and $k_{2}$ of $\nu_{1}$ and $\nu_{2}$ flux from the sun, respectively, as defined in Sec. 2. Therefore

$$
k_{1}=\frac{1}{2}+C_{0}, k_{2}=\frac{1}{2}-C_{0} .
$$

Finally, we transform to the $\nu_{e}-\nu_{x}$ basis, so

$$
\rho^{\nu_{e} \nu_{x}}=U\left(\theta_{V}\right) \rho U^{\dagger}\left(\theta_{V}\right) \equiv\left(\begin{array}{cc}
\rho_{e e} & \rho_{e x} \\
\rho_{x e} & \rho_{x x}
\end{array}\right) .
$$


One then finds that the probability of observing a neutrino reaching the surface of the earth as an electron neutrino is given by

$$
P_{S}=\rho_{e e}=\frac{1}{2}+C_{0} \cos 2 \theta_{V} .
$$

The off-diagonal matrix element is given by

$$
\rho_{x e}=-C_{0} \sin 2 \theta_{V}
$$

Our numerical simulations were all performed by integrating Eq. (A.5) to solve for $P_{2 e}$, and also by integrating the density matrix equations of motion. The evolution of the density matrix is given by

$$
i \hbar \partial_{t} \rho=-[\rho, H]
$$

Using Eq. (A.36) with the Hamiltonian in the flavor basis, we find that our new equations of motion are

$$
\begin{gathered}
i \partial_{t} \rho_{e e}=A\left(\rho_{x e}-\rho_{x e}^{*}\right) \\
i \partial_{t} \rho_{x e}=2\left(A \rho_{e e}-B \rho_{x e}\right)-A,
\end{gathered}
$$

where $A$ and $B$ are defined in Eqs. (A.7) and (A.8). This allows us to perform calculations using the complete mixed ensemble. The expressions given in Eqs. (A.34) and (A.35) form a steady state solution of the density matrix equations of motion in the vacuum. For typical $\left(\Delta m^{2} \approx 1 \times 10^{-5} \mathrm{eV}^{2}\right.$ and $\left.E=8 \mathrm{MeV}\right)$ maximally mixed Boron- $8\left({ }^{8} B\right)$ neutrinos, $P_{2 e}$ oscillates and can take any value between 0 and 1. $C_{0} \approx-\frac{1}{2}$, which means that $k_{2} \approx 1$, and Eq. (2.4) reduces to $P_{S E}=P_{2 e}$. Thus $P_{S E}$ exhibits oscillatory behavior, and is in no way constrained to be $1 / 2$ at maximal mixing.

\section{B. Calculation methodology for the day-night effect}

First we calculated $P_{S}$ using Eq. (A.34) for the spectrum of $\Delta m^{2} / p$ at various mixing angles. For a given $\Delta m^{2} / p$ we averaged $P_{S}$ over the regions of ${ }^{8} B$ neutrino production in the sun, provided by Ref. [33]. Using $P_{S}$ to describe the neutrinos that arrive at the earth, we then performed the evolution through the earth with the density matrix equations of motion. The initial conditions for the density matrix are given by

$$
\rho_{e e}=P_{S}
$$

and

$$
\rho_{x e}=-\frac{1}{2}\left(2 P_{S}-1\right) \tan 2 \theta_{V}
$$

At maximal mixing we assume that $\rho_{x e}=-\frac{1}{2} \cos \left(2 \theta_{M}\left(t_{0}\right)\right) \sin 2 \theta_{V} \approx \frac{1}{2}$ which is the adiabatic result. This assumption is justified because in the regions of parameter 


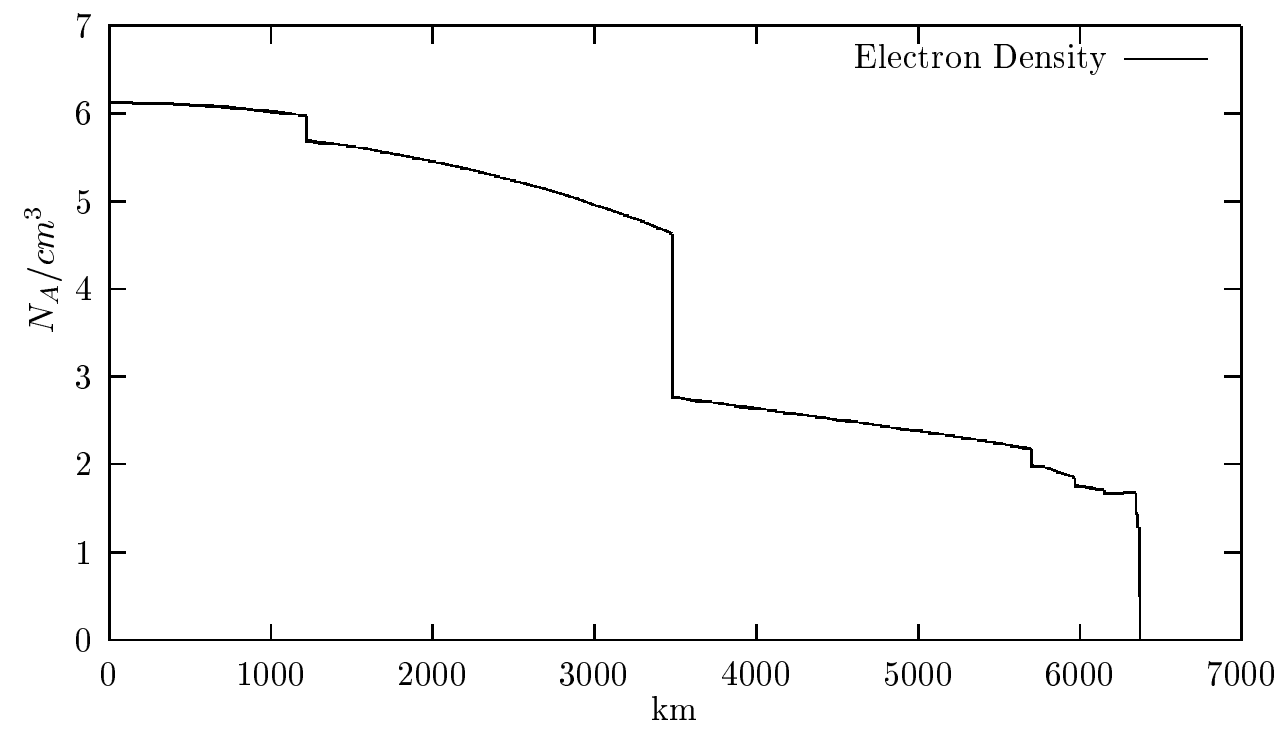

Figure 5: The Preliminary Reference Earth Model (PREM) electron density $\left(N_{e}\right)$ profile of the earth. $N_{e}$ is shown in units of Avogadro's number of electrons per $\mathrm{cm}^{3}$.

space under consideration near maximal mixing, $P_{\text {jump }} \approx 0$. It follows that in these same regions of parameter space the evolution remains adiabatic in the limit where $\theta_{V}=\pi / 4$. We use the earth density profile given in the Preliminary Reference Earth Model (PREM) [34] (see Fig 5). To convert from the mass density to electron number density we use the charge to nucleon ratio $Z / A=0.497$ for the mantle and $Z / A=0.467$ for the core. The numerical calculations were performed using a fourth order Runge-Kutta integration programmed in $\mathrm{C}++$. We propagated the neutrinos through the earth for 90 zenith angles, $\alpha$, evenly spaced between 90 and 180 degrees. We calculate the anticipated electron flux as a function of zenith angle and energy, denoted $P_{S E}\left(\alpha, E_{\nu}\right)$. The calculation parameters are chosen for those of the Super-Kamiokande detector. The normalized ${ }^{8} B$ neutrino spectrum, $\Phi\left(E_{\nu}\right)$, and solar electron densities, $N_{e}$, are also obtained from data-files provided by Ref. [33]. Effective neutrino cross sections are available which take into account the electron recoil cross section with radiative corrections, the energy resolution, and the trigger efficiency [35, 33]. We used these more accurate cross sections for the overall daynight effect plotted in Fig. 2. Because these effective cross sections already include the integration over detected electron recoil energy, to calculate the recoil electron spectrum we used the differential neutrino-electron scattering cross sections given in Ref. [36]. Using these data files and numerical results the cross section for the scattering of solar neutrinos of energy $E_{\nu}$ with electrons to produce a recoil electron of energy $T^{\prime}$ at the zenith angle $\alpha$ is given by

$$
\frac{d \sigma_{\nu_{\text {solar }}}}{d T^{\prime}}\left(T^{\prime}, E_{\nu}, \alpha\right)=P_{S E}\left(\alpha, E_{\nu}\right) \frac{d \sigma_{\nu_{e}}}{d T^{\prime}}\left(T^{\prime}, E_{\nu}\right)+\left[1-P_{S E}\left(\alpha, E_{\nu}\right)\right] \frac{d \sigma_{\nu_{\mu}}}{d T^{\prime}}\left(T^{\prime}, E_{\nu}\right) .
$$

Since muon and tau neutrinos have the same neutral current interactions, we can use 
the $\nu_{\mu}$ cross section for the $\nu_{x}$. The analysis of the recoil electron spectra is explained in Refs. [35. The actual flux at recoil energy, $T$, is

$$
g(\alpha, T)=\int_{0}^{\infty} d E_{\nu} \Phi\left(E_{\nu}\right) \int_{0}^{T_{\max }^{\prime}} d T^{\prime} R\left(T, T^{\prime}\right) \frac{d \sigma_{\nu_{\text {solar }}}}{d T^{\prime}}\left(T^{\prime}, E_{\nu}, \alpha\right)
$$

where the energy resolution of the detector is incorporated through

$$
R\left(T, T^{\prime}\right)=\frac{1}{\Delta_{T^{\prime}} \sqrt{2 \pi}} \exp \left(\frac{-\left(T^{\prime}-T\right)^{2}}{2 \Delta_{T^{\prime}}^{2}}\right)
$$

The energy resolution, $\Delta_{T^{\prime}}$, around the true electron energy $T^{\prime}$ for Super-Kamiokande is given by

$$
\Delta_{T^{\prime}}=(1.6 \mathrm{MeV}) \sqrt{T^{\prime} /(10 \mathrm{MeV})} .
$$

To calculate the average day-night effect over one year, we weight the flux by the zenith angle exposure function $Y(\alpha)$ explained in Appendix D. The daytime measured flux at a given measured electron recoil energy, $T$, is given by

$$
D(T)=\int_{0}^{90} d \alpha g(\alpha, T) Y(\alpha)
$$

and for nighttime is

$$
N(T)=\int_{90}^{180} d \alpha g(\alpha, T) Y(\alpha) .
$$

The day-night asymmetry as a function of recoil electron energy plotted in Fig. 4 is given by

$$
A_{d-n}(T)=\frac{N(T)-D(T)}{N(T)+D(T)}
$$

The final day-night asymmetry plotted in Fig. 2 is given by

$$
A_{d-n}=\frac{\int_{5 \mathrm{MeV}}^{\infty} d T(N(T)-D(T))}{\int_{5 \mathrm{MeV}}^{\infty} d T(N(T)+D(T))}
$$

where $5 \mathrm{MeV}$ is the minimum energy detected at Super-Kamiokande. Verification of the accuracy of the computer code has been accomplished with the help of [37], and by comparing our simulations to plots and data available in the literature.

\section{Validity of the steady state approximation}

Most of the work in the past decade on the MSW effect has assumed that the ensemble of neutrinos reach the earth in a steady state solution of the density matrix (i.e., in an incoherent mixture of the mass eigenstates $\nu_{1}$ and $\nu_{2}$ ). There are several reasons that the neutrinos reach the earth in a steady state: (a) The separation of the $\left|\nu_{1}\right\rangle$ and $\left|\nu_{2}\right\rangle$ wave-packets while propagating from the sun to the earth exceeds the size of 
the individual wave packets, eliminating the interference effects. (b) The eccentricity of the earth's orbit results in a daily change of the earth-sun radius larger than the vacuum oscillation length of the neutrinos. (c) The neutrinos are produced in a region much larger than their local oscillation length. (d) The energy resolution of the current detectors coupled with the earth-sun radius perform an average. We now proceed to map out parameter space justifying where the steady state approximation is valid. First we consider the separation of the two eigenstates during transit to the earth. This results in system that is an incoherent superposition of $\left|\nu_{1}\right\rangle$ and $\left|\nu_{2}\right\rangle$. The width of the wave-packets, $\sigma_{x}$, is given by Ref. [38:

$$
\sigma_{x} \approx 0.9 \times 10^{-7} \mathrm{~cm} .
$$

This results in a coherence length given by:

$$
L_{c o h}=2 \sqrt{2} \sigma_{x} \frac{2 E^{2}}{\Delta m^{2}} .
$$

We lose coherence between the mass eigenstates if $L_{\text {coh }}<1 \mathrm{AU}=1.5 \times 10^{13} \mathrm{~cm}$. If we require that the incoherence condition apply up to $14 \mathrm{MeV}$ to include all ${ }^{8} \mathrm{~B}$ neutrinos, we find that for all of $\sin ^{2} 2 \theta_{V}$ where $\Delta m^{2}>6.63 \times 10^{-6} \mathrm{eV}^{2}$ the wavepackets have separated upon reaching earth. This corresponds to the region labeled (a) in Fig. 6. Because there is a continuous beam of neutrinos arriving from the sun, we can ignore the fact that the lighter mass eigenstate arrives first, and simply drop terms that rapidly oscillate due to the lack of interference between the two states.

In the previous case the interference effects vanish because of a loss of coherence between the mass eigenstates for a neutrino produced at a specific place and time. In the remaining topics the interference effects vanish due to averaging over the ensemble of neutrinos which reach the detector. Regardless of the source of the incoherence, any two systems with the same density matrix behave identically in single counting experiments [39.

Next, we analyze the effect of the eccentricity of the earth's orbit . We are interested in day-night effects; therefore, if the earth-sun radius changes more than an oscillation length during one day, this will result in washing out any phase dependence in the results measured over a period of one year. Between perihelion and aphelion the earth-sun radius changes $2 e(1 \mathrm{AU})=5.1 \times 10^{11} \mathrm{~cm}$, where $e=0.017$ is the earth eccentricity. The earth-sun radius changes by this quantity once each 180 days giving an average daily change in radius of $2.83 \times 10^{9} \mathrm{~cm}$. This ensures our incoherent phase for $\Delta m^{2}>1.2 \times 10^{-6} \mathrm{eV}^{2}$. This region is denoted by everything above the line marked (b) in Fig. 6.

Third, we study the impact of where the neutrinos were produced. If the neutrino region of production is greater than the local oscillation length of the neutrinos, then neutrinos of all possible phases exist in the ensemble. For a continuous beam of neutrinos, this also results in dropping the rapidly oscillating terms. The condition 
is satisfied for the entire parameter space under consideration $0.001<\sin ^{2} 2 \theta_{V}<1$ and $1 \times 10^{-11} \mathrm{eV}^{2}<\Delta m^{2}<1 \times 10^{-3} \mathrm{eV}^{2}$. However, one must be careful in making this statement. Although the region of production may be greater than the neutrino oscillation length in the sun, the neutrinos could undergo a non-adiabatic transition bringing a specific phase into dominance. This is the case for vacuum oscillations $\left(\Delta m^{2} \approx 4 \times 10^{-10} \mathrm{eV}^{2}\right)$. The ${ }^{8} B$ neutrinos are produced mostly at $R_{8_{B}}=0.046 R_{\text {sun }}=3.2 \times 10^{9} \mathrm{~cm}$. The vacuum oscillation length is on the order of $1 \mathrm{AU}$. However the oscillations length near the solar core where these neutrinos are produced is about $1.8 \times 10^{7} \mathrm{~cm} \ll R_{8_{B}}$. Although the neutrinos are produced in a region larger than their oscillation length, they acquire roughly the same phase in the process of leaving the sun. This occurs because the density change upon leaving the sun occurs more rapidly than the oscillation length of the neutrinos, violating the condition of adiabaticity. To express this quantitatively we estimate that if $P_{\text {jump }}<0.1$ for $14 \mathrm{MeV}$ neutrinos that the initial randomly distributed oscillation phases at the time of production will persist as the neutrinos leave the sun and enter the vacuum. This leads to a steady state solution applicable in the parameter space above the diagonal line labeled (c) shown in Fig. 6.

Last, we study the impact of the energy resolution on our ability to discriminate phases. Assuming perfect coherence between the two mass eigenstates the phase upon reaching the earth is given by

$$
\phi=\frac{\Delta m^{2}(1 \mathrm{AU})}{4 p \hbar c} .
$$

Our uncertainty in energy impacts our uncertainty in phase through error propagation:

$$
\delta \phi=\left|\frac{d \phi}{d p}\right| \delta p=\frac{\Delta m^{2}(1 \mathrm{AU})}{4 p^{2} \hbar c} \delta p .
$$

If our uncertainty in our phase is greater than $2 \pi$ we are again justified in treating our ensemble as a steady state. Using conservative figures for energy $(p=14 \mathrm{MeV})$, and the energy resolution $(\delta p \approx 1 \mathrm{MeV})$ [35], we find that for $\Delta m^{2}>6.5 \times 10^{-9} \mathrm{eV}^{2}$ we are justified in the steady state approximation. This corresponds to parameter space above the line labeled (d) in Fig. 6. Recently Ref. [21] also reached the same conclusions outlined in this appendix.

\section{The zenith distribution function}

The zenith angle distribution function gives the fraction of the time that the sun is at a given zenith angle. The function is calculated by numerically simulating the orbit of the earth around the sun. We begin by writing the vector towards the zenith 


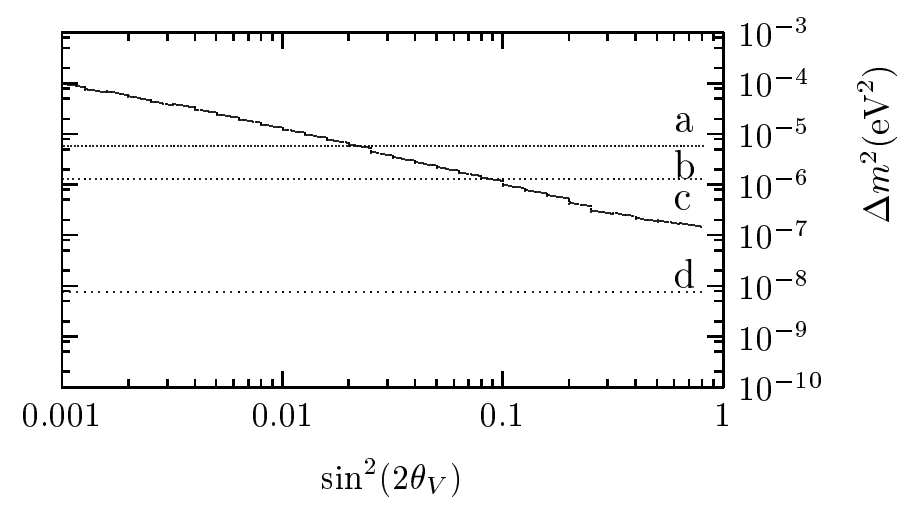

Figure 6: The regions satisfying the conditions for steady state density matrix. Above the line (a) is in steady state because of wave packet separation. Above the line (b) can be treated as steady state because of the eccentricity of the earth's orbit. Above the diagonal line (c) is in steady state because the region producing the neutrinos is much larger than an oscillation length, and this phase averaging survives until the neutrinos reach the vacuum. Above line (d) is in steady steady state because of the energy resolution of our detectors.

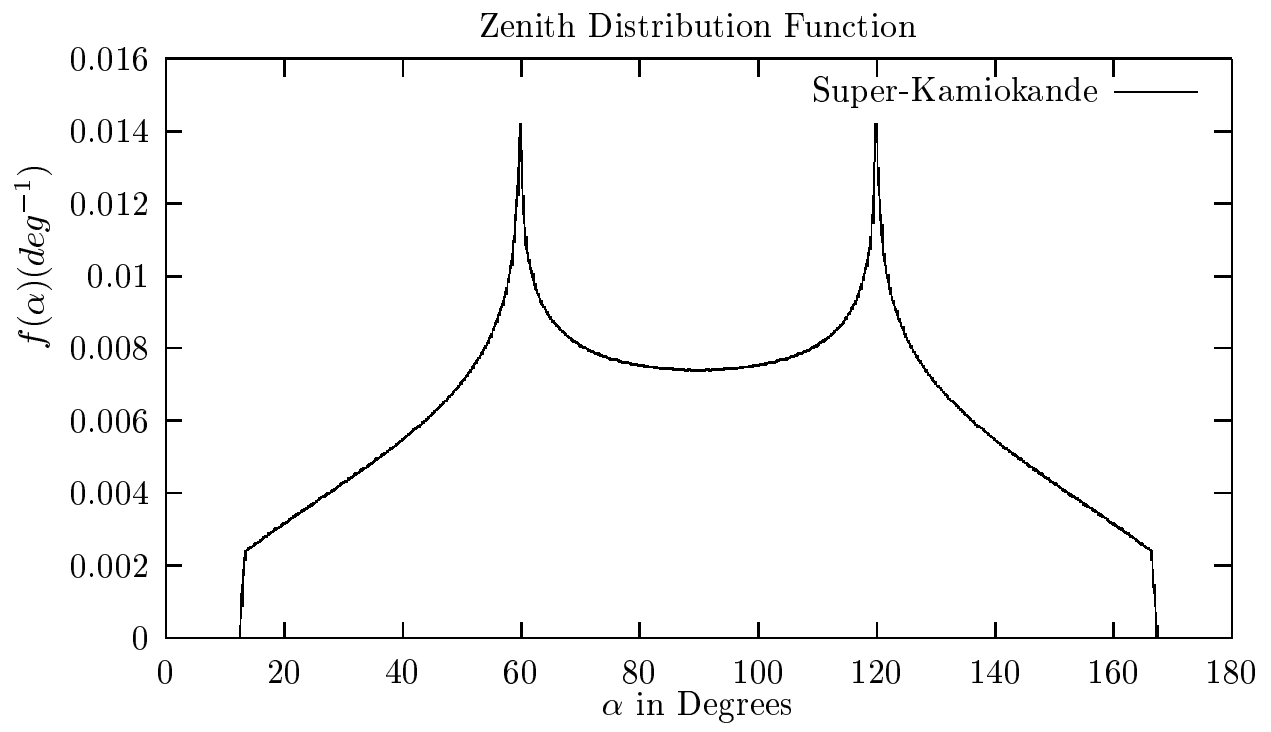

Figure 7: The zenith distribution function at Super-Kamiokande.

of the detector in coordinates for which the earth's orbit lies in the $x$ - $y$ plane:

$$
\vec{r}_{z}=\left(\begin{array}{ccc}
1 & 0 & 0 \\
0 & \cos \delta & -\sin \delta \\
0 & \sin \delta & \cos \delta
\end{array}\right)\left(\begin{array}{c}
\sin \left(90^{\circ}-L\right) \cos \phi \\
\sin \left(90^{\circ}-L\right) \sin \phi \\
\cos \left(90^{\circ}-L\right)
\end{array}\right)
$$

where the north latitude is given by $L, \phi$ gives the time of day in radians, and $\delta=23.439^{\circ}$ is the earth's declination [40]. Because we are averaging over a one year 
time period we can arbitrarily choose the initial time of year, and the initial time of day. The vector pointing from the earth towards the sun is

$$
\vec{r}_{s}=\left(\begin{array}{c}
\cos D \\
\sin D \\
0
\end{array}\right)
$$

where $D$ is the day of the year in radians. From here we can find the local zenith angle from the dot product $\vec{r}_{s} \cdot \vec{r}_{z}=\cos \alpha$. To numerically calculate the zenith function distribution we divided $\alpha$ into 360 bins evenly spaced between 0 and $\pi$. Now we run $0 \leq D \leq 2 \pi$ and $0 \leq \phi \leq 2 \pi$ over 1000 steps in $D$ and 1000 steps in $\phi$ and count how much relative time $\alpha$ spends over each bin. We generate the zenith angle distribution function for Super-Kamiokande which sits in Gifu Prefecture, Japan at $36.43^{\circ}$ north latitude [19]. This produces the undistorted zenith function distribution seen in Fig. 7. One can also obtain this function as a data file from [33] which includes small corrections for the eccentricity of the earth's orbit and the wobble of the earth's declination. To maximize accuracy we performed our calculations using this data file.

\section{References}

[1] H. Fritzsch and Z. Z. Xing, Lepton mass hierarchy and neutrino oscillations, Phys. Lett. B 372 (1996) 265.

[2] V. Barger, S. Pakvasa, T. Weiler, and K. Whisnant, Bi-maximal mixing of three neutrinos, Phys. Lett. B 437 (1998) 107. hep-ph/9806387.

[3] A. J. Baltz, A. S. Goldhaber, and M. Goldhaber, The solar neutrino puzzle; an oscillation solution with maximal neutrino mixing, Phys. Rev. Lett. 81 (1998) 5730. hep-ph/9806540.

[4] Y. Nomura and T. Yanagida, Bi-maximal neutrino mixing in SO(10) GUT, Phys. Rev. D 59 (1999) 017303. hep-ph/9807325.

[5] H. Fritzsch and Z. Z. Xing, Large leptonic flavor mixing and the mass spectrum of leptons, Phys. Lett. B 440 (1998) 313. hep-ph/9808272.

[6] S. Davidson and S. King, Bi-maximal neutrino mixing in the MSSM with a single right-handed neutrino, Phys. Lett. B 445 (1999) 191. hep-ph/9808296.

[7] S. K. Kang and C. S. Kim, Bi-maximal lepton flavor mixing matrix and neutrino oscillation, Phys. Rev. D 59 (1999) 091302. hep-ph/9811379.

[8] L. Wolfenstein, Neutrino oscillations in matter, Phys. Rev. D 17 (1978) 2369.

[9] S. P. Mikheyev and A. Y. Smirnov Soviet Journal of Nuclear Physics 42 (1985) 913. 
[10] S. P. Mikheyev and A. Y. Smirnov Nuovo Cimento C 9 (1986) 17.

[11] V. Barger and K. Whisnant, Seasonal and energy dependence of solar neutrino vacuum oscillations. hep-ph/9903262.

[12] E. D. Carlson, Terrestrially enhanced neutrino oscillations, Phys. Rev. D 34 (1986) 1454.

[13] A. J. Baltz and J. Weneser, Effect of transmission through the earth on neutrino oscillations, Phys. Rev. D 35 (1987) 3364.

[14] A. J. Baltz and J. Weneser, Implication of gallium results on the possibility of observing day-night matter oscillations at SNO Super-Kamiokande and BOREXINO, Phys. Rev. D 50 (1994) 5971.

[15] E. Lisi and D. Montanino, Earth regeneration effect in solar neutrino oscillations: an analytic approach, Phys. Rev. D 56 (1997) 1792. hep-ph/9702343.

[16] Q. Y. Liu, M. Maris, and S. T. Petcov, A study of the day night effect for the Super-Kamiokande I: Time averaged solar neutrino probability, Phys. Rev. D 56 (1997) 5991.

[17] M. Maris and S. Petcov, A study of the day-night effect for the Super-Kamiokande detector: II. electron spectrum deformations and day-night asymmetries, Phys. Rev. D 56 (1997) 7444. hep-ph/9705392.

[18] J. N. Bahcall and P. I. Krastev, Does the sun appear brighter at night in neutrinos?, Phys. Rev. C 56 (1997) 2839. hep-ph/9706239.

[19] Y. Fukuda and The Super-Kamiokande Collaboration, Constraints on neutrino oscillation parameters from the measurement of day-night solar neutrino fluxes at Super-Kamiokande, Phys. Rev. Lett. 82 (1999) 1810. hep-ex/9812009.

[20] S. P. Mikheyev and A. Y. Smirnov, Resonance oscillations of neutrinos in matter, Soviet Physics - USPEKHI 30 (1987) 759.

[21] A. Dighe, Q. Liu, and A. Y. Smirnov, Coherence and the day-night asymmetry in the solar neutrino flux. hep-ph/9903329.

[22] S. Petcov, Diffractive-like (or parametric-resonance-like?) enhancement of the earth (day-night) effect for solar neutrinos crossing the earth core, Phys. Lett. B 434 (1998) 321. hep-ph/9805262.

[23] S. Petcov, The oscillation length resonance in the transition of solar and atmospheric neutrinos crossing the earth core. hep-ph/9811205.

[24] E. Akhmedov, Remarks on parametric resonance of neutrino oscillations in the earth. hep-ph/9903302. 
[25] C. Giunti, Is bi-maximal mixing compatible with the large angle msw solution of the solar neutrino problem?, Phys. Rev. D 59 (1999) 077301. hep-ph/9810272.

[26] J. N. Bahcall, P. I. Krastev, and A. Y. Smirnov, Where do we stand with solar neutrino oscillations?, Phys. Rev. D 58 (1998) 096016. hep-ph/9807216.

[27] R. Barbieri et al., Oscillations of solar and atmospheric neutrinos, JHEP 12 (1998) 017. hep-ph/9807235.

[28] J. N. Bahcall, S. Basu, and M. Pinsonneault, How uncertain are solar neutrino predictions?, Phys. Lett. B 433 (1998) 1.

[29] The CHOOZ Collaboration (M. Apollonio et al.), Initial results from the CHOOZ long baseline reactor neutrino oscillation experiment, Phys. Lett. B 420 (1998) 397. hep-ex/9711002.

[30] S. Parke, Nonadiabatic level crossing in resonant neutrino oscillations, Phys. Rev. Lett. 57 (1986) 1275.

[31] A. B. Balantekin, Exact solutions for matter-enhanced neutrino oscillations, Phys. Rev. D 58 (1998) 013001. hep-ph/9712304.

[32] A. B. Balantekin and J. F. Beacom, Semiclassical treatment of matter-enhanced neutrino oscillations for an arbitrary density profile, Phys. Rev. D 54 (1996) 6323. hep-ph/9606353.

[33] J. N. Bahcall, 1998. http://www.sns.ias.edu/jnb/.

[34] A. M. Dziewonski and D. L. Anderson, Preliminary reference earth model, Physics of the Earth and Planetary Interiors (1981).

[35] J. N. Bahcall, P. Krastev, and E. Lisi, Neutrino oscillations and moments of electron spectra, Phys. Rev. C 55 (1997) 494. nucl-ex/9610010.

[36] J. N. Bahcall, Neutrino Astrophysics. Cambridge University Press, 1989.

[37] P. I. Krastev, 1999. Private Communications.

[38] C. W. Kim and A. Pevsner, Neutrinos in Physics and Astrophysics. Harwood Academic Publishers, 1993.

[39] L. Stodolsky, When the wavepacket is unnecessary, Phys. Rev. D 58 (1998) 036006.

[40] The Astronomical Almanac. US Government Printing Office, 1996. 